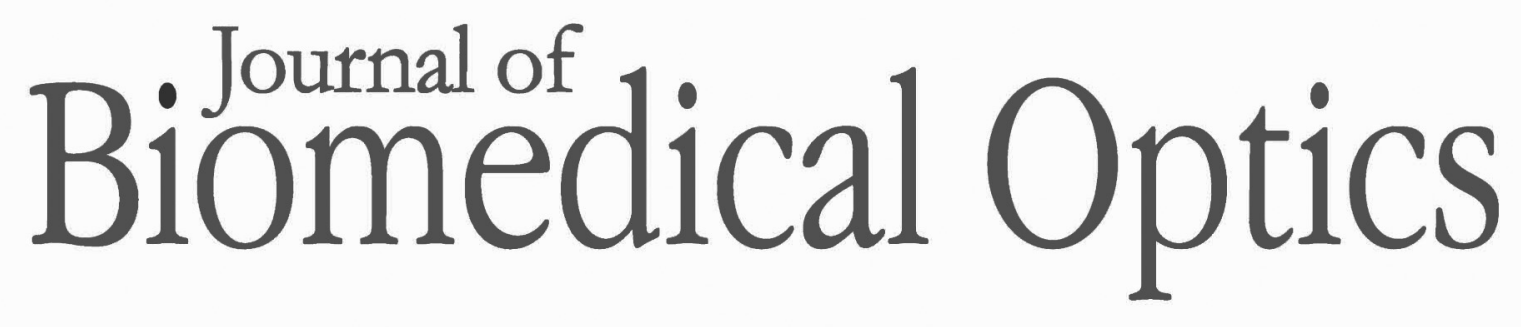

SPIEDigitalLibrary.org/jbo

\title{
Molecular Fluorescence: Principles and Applications, Second Edition
}

Barry R. Masters 


\section{BOOK REVIEW}

\section{Molecular Fluorescence: Principles and Applications, Second Edition}

Bernard Valeur and Mário N. Berberan-Santos, 569 pages + xxii, ISBN: 978-3-527-32837-0, Wiley-VCH, Weinheim, Germany (2012), \$185.00, hardcover.

Reviewed by Barry R. Masters, Visiting Scientist, Department of Biological Engineering, Massachusetts Institute of Technology. Fellow of AAAS, OSA, and SPIE. E-mail: bmasters@mit.edu.

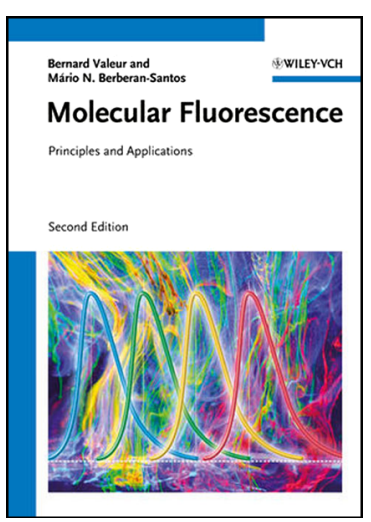

Why do I highly recommend Molecular Fluorescence: Principles and Applications, Second Edition to the readers of the Journal of Biomedical Optics? While there are many books that purport to elucidate the principles of fluorescence spectroscopy and discuss the instrumentation and the plethora of applications of fluorescence techniques, this book is unique in its depth of physical and mathematical exposition.

While qualitative explanations of molecular fluorescence can only serve to introduce the subject, a deeper understanding of the subject must include a clear presentation of the physics which forms the foundation of the physical phenomena and its host of wonderful applications. The authors have achieved a difficult task: they present the physics at a rigorous level that is physically and mathematically correct to a broad interdisciplinary audience that may not possess a strong background in quantum mechanics, group theory, and molecular orbital theory. My perusal of this book supports my premise that the authors eminently succeeded in their task.

Molecular Fluorescence begins with a chapter on the historical development of luminescence. The authors also provide a general bibliography of monographs and books. The astute reader may rightfully inquire: what is the value of a historical description of a modern, rapidly developing field of science? First there is no substitute for reading the original papers of the development of a field of science. While many of the early papers in physics are written in French, German, or Russian languages, there are translations available. A person who begins the study of a discipline of science should be familiar with the primary literature, because in contrast to the secondary literature, the primary literature usually provides a better analysis of the physics and the mathematical description. Moreover, there is usually a more complete description of the limitations of the theory, and the techniques that are often truncated or omitted in secondary literature which purports to summarize the original papers. An author will often cite the original papers of a field in their references, but in fact they have never read the original papers. It is clear to me that the authors of Molecular Fluorescence have read and digested all of the papers that they cited in their book. Second, if the reader of Molecular Fluorescence intends to teach this subject to students, it is crucial to understand the contributions of a disparate group of scientists. To exclude the contributions of a group of scientists because they published in the Russian scientific literature or in another foreign language is both unethical and not consistent with scholarly standards of publications.

Molecular Fluorescence succeeds as a unique exposition of the field due to several innovative pedagogical techniques. The references appear within the chapters, which makes it easy to locate the key sources and associate them with the text. The excellent diagrams, many of which are in color, are integrated with the text and help the reader to understand the physics, the mathematical description, the kinetics of the processes, and the instruments. Furthermore, the authors show the molecular structure of many of the molecules that are described in the text. The physical phenomena of luminescence is completely integrated with the chemical structures and their electronic and vibrational states so that that the reader will not only learn what occurs in a particular process, but the how and the why in terms of a classical or a quantum mechanical description. The reader is aided by an appendix that details the characteristics of fluorescent organic compounds, as well as a detailed index.

Further evidence of unique exposition is found in the chapter "Excitation Energy Transfer." I know of no other textbook or reference book that integrates the various mechanisms of energy transfer into a cohesive detailed exposition. In addition to presenting a succinct version of both the classical and the quantum mechanical theory of FRET, the authors include a clear description of the various classes of coupling in terms of strong coupling, weak coupling, and very weak coupling [the regime of Förster's resonance energy transfer (FRET)]. Often publications invoke the theory of FRET in experimental situations that fail to support the inherent assumption of FRET; these events could be less frequent if the authors carefully studied the original papers of Förster and the alternative theories of energy transfer that are cited in Molecular Fluorescence.

Mechanisms of energy transfer are relatively easy to implement in the laboratory; in fact, there are popular computer programs that take the spectra of the donor-acceptor pairs and yield plots of the derived perimeters such as separation distances. But without a critical understanding of the assumptions of an individual theory of an energy transfer mechanism, the derived outputs are deeply confounded, and false conclusions and interpretation of the data are prevalent. This example serves to illustrate the importance of understanding the assumption of each theory, each experimental method, and the required subsequent validation of the theoretical assumptions and their relevance to 
the experimental conditions. I think that the chapters contained within Molecular Fluorescence contain the prescribed critical description of the physics, chemistry, and instrumentation of luminescence that helps the reader to avoid the common errors of the misuse of the theory and applications of luminescence techniques. The balance between theory and application is excellent. The second edition presents many of the modern fascinating and useful applications of molecular spectroscopy on the scales of single molecules, molecular sensors, molecular structures, tissues, organs, and whole organisms, and all of these developments greatly support fundamental scientific research and medical applications. Molecular Fluorescence is a comprehensive textbook and reference book that provides a deep and critical analysis of this evolving field. 